\title{
British Association for Accident and Emergency Medicine, Cardiff, 15-18 April 1997
}

Shootings in Liverpool: a little local trouble or a nascent epidemic? U Geary, L C Luke, H Morris, D A Ritchie Department of Accident and Emergency Medicine, Royal Liverpool University Hospital, Prescot Street, Liverpool

A recent spate of gangland warfare, prompted by territorial claims and drug merchandising, led to a dramatic escalation in the number of victims of shootings admitted to the accident and Emergency department of the Royal Liverpool University Hospital. In a recent 30 month period, 40 victims of missile injuries were treated; this represented a $1000 \%$ increase in the caseload since the early 1990s. The direct mortality following shooting was five $(12 \%)$ with one additional delayed death; the age range of the victims was 17-49 years with a mean age of 27 years and the mean length of hospital admission was $14 \mathrm{~d}$. There was very considerable morbidity among the survivors, especially with severe limb injuries and psychological disorders. The male:female ratio among the victims was 12.6 . In this paper we describe and illustrate the caseload from gun related violence at the hospital and outline the diagnostic and management implications. The impact of this epidemic on the hospital was very substantial in terms of the use of resources and security issues. We highlight the lessons we have learned from our experience; in particular we address the frequently unexpected nature of victims' arrival at the hospital, the confounding factors of substance misuse and criminality, and the need for an accelerated and coherent multi disciplinary trauma response.

Trolley time-is it an accurate or reliable performance indicator?

J Edhouse, N Kidner, J Wardrope

Accident and Emergency Department, Northern General Hospital, Heries Road, Sheffield

The Department of Health intends to use waiting time for a hospital bed as a measure of the performance of A\&E departments. Waiting time is defined as the interval between decision to admit and the time a patient receives a ward bed; the Patient's Charter standard is $2 \mathrm{~h}$. All A\&E departments in England and Wales seeing >15000 new patients/ year were surveyed by postal questionnaire. Data from 70 departments were studied $25 / 70$ do not measure waiting time according to the standard definition; 30/70 responders feel that $>80 \%$ of their timings were inaccurately recorded; 18 departments are storing handwritten data and retrieving it manually. Forty five departments were used to compare admissions procedures and performance according to the Patient's Charter standard. For the purpose of this study, departments with waiting time for a hospital bed $<2$ hours in at least $90 \%$ of patients, were classed as "good performers" (27/45), and the remainder were classed as "average performers" (18/45); $35 / 45$ use bed managers and admissions units/assessment wards for the majority of admissions. "Average performance" appears to be related to department size (>50 000/ year); using A\&E for GP admissions also led to poor performance. Waiting time for a hospital bed, as presently defined, is a good way to measure the efficiency with which a hospital processes its emergency admissions, but does not provide a true reflection the performance of the A\&E department. There appear to be relatively long waits on trolleys in larger departments, despite their having bed managers and admissions units in place. The total length of time spent on a trolley (A\&E time plus hospital wait time) is a good measure of hospital performance; the data obtained are likely to be more accurate, and are already collected routinely in all $\mathrm{A} \& \mathrm{E}$ departments.

\section{Chest pain in the emergency} department: potential impact of structured history taking and computer decision support

S Mason, R L Kennedy, R F Harrison, A M Burton, J Wardrope

Accident and Emergency Department, Northern General Hospital, Herries Road, Sheffield

Early diagnosis of acute myocardial infarction (AMI) in patients with acute chest pain is notoriously difficult. In this study, we assessed the effect a structured history from, and the potential of, a computer algorithm on emergency room diagnosis and triage. Phase I of the study involved follow up of 397 consecutive attendances with non-traumatic chest pain (91 AMI, 133 angina, 172 non-cardiac). In phase II, history and examination details were entered on a purpose designed form (127 patients: 27 AMI, 64 angina, 36 non-cardiac). In phase III, clinical details were entered into a computer in the emergency department (139 patients: 29 AMI, 53 angina, 57 non-cardiac). These data were fed into an artificial neural network (ANN) trained, and previously validated, to diagnose AMI. In phase I, 9/305 (2.9\%) were wrongly diagnosed as AMI and 32/91 AMIs were not accurately identified. Only two patients were discharged in error (one unstable angina and one AMI) but 40 patients who could safely have been discharged were admitted. Structured history and examination in phase II had no impact on the false positive diagnosis rate $(2.7 \%)$ or on the proportion of AMIs not accurately identified in the emergency room (34.5\%). In phase III, the ANN diagnosed six AMIs which were incorrectly diagnosed by the doctors. In conclusion, early identification and confident exclusion of AMI in the emergency room is difficult. There is clear scope for improving diagnosis and triage of chest pain patients. Structured history taking alone does not improve performance of junior doctors in the emergency department, although documentation of the cases was improved. Preliminary study of an ANN system showed the feasibility of data entry in the emergency department and potential for such decision support to improve clinical practice.

Which asthmatics in A\&E need blood gases?

B D W Harrison, A Cooper

Department of Respiratory Medicine, Accident and Emergency Department, Norfolk and Norwich Hospital, Brunswich Road, Norwich

Background-The new British Thoracic Society (BTS) guidelines for the management of inpatients with asthma recommend measurement of arterial blood gases (ABG) only on patients who either have oxygen saturations less than $92 \%$ or who have any life threatening features. The guidelines for asthma in $A \& E$ departments have not been amended and still recommend ABG on all asthma patients with a peak expiratory flow (PEF) less than $50 \%$ of best.

Objective-To assess whether the new BTS guidelines for the measurement of ABG on asthmatic inpatients are also applicable to patients presenting to $A \& E$ with an exacerbation of asthma.

Methods-The subjects were previously diagnosed asthmatics aged 18-64 years presenting to A\&E with a PEF less than $75 \%$ of their best value. The presenting PEF\% (PEF/best PEF) was noted as a marker of severity of attack. ABG and oxygen saturation measured by pulse oximetry $\left(\mathrm{SpO}_{2}\right)$ were simultaneously recorded on all patients. Those patients who had respiratory failure (defined by $\mathrm{PaCO}_{2}>6$ $\mathrm{kPa}$ and $/ \mathrm{or} \mathrm{PaO}_{2}<8 \mathrm{kPa}$ ) and those requiring ventilation were identified.

Results-ABG were recorded on 66 subjects ( 35 female, 31 male). 20 patients had presenting $\mathrm{PEF} \%$ of $33-50 \%$ and 22 patients presented with $\mathrm{PEF} \%<33 \%$. Three patients had respiratory failure. All presented with clinical features of a life threatening attack of asthma. The two patients who required ventilation both presented with $\mathrm{Spo}_{2}<92 \%$ despite maximum oxygen treatment. All other patients responded to medical treatment.

Conclusions-The current BTS guidelines for the measurement of blood gases on inpatients with asthma can safely be extended to A\&E. Blood gases need only be recorded on patients with saturations $<92 \%$ despite supplemental $\mathrm{O}_{2}$, those patients with features of a life threatening attack of asthma, and those patients who are not responding to treatment. 
The ability of accident and emergency department SHOs to diagnose ulnar collateral ligament (UCL) injuries of the thumb in an urban population M Jones, C Muwanga, J Stephenson

Department of Accident and Emergency, Sunderland District General Hospital, Kayll Road, Sunderland, Tyne and Wear

Background-Hand injuries account for $21 \%$ of injuries presenting to the A\&E department in Sunderland. Early diagnosis of ruptured UCL and repair offers the best outcome. It is therefore important that such injuries are diagnosed early and referred to the appropriate specialist.

Aims-To assess the mechanism of injury, the initial clinical diagnosis and timing of referral to the hand specialty service, comparing the definitive operative findings with these results Methods-The information was obtained by a retrospective analysis of notes documenting basic demographic data, mechanism of injury, times to presentation and referral, initial clinical diagnosis, and the final operative diagnosis, from 70 patients with a confirmed UCL rupture at surgery over a period of five years. Results-The commonest cause of injury was related to non-sporting activities, the majority due to falls, assaults, and injuries in the workplace. Of concern, the A\&E SHOs correctly identified ruptures of the thumb UCL in around only a third of the cases.

Conclusions-Despite a formal teaching programme on hand injuries with particular emphasis on injuries to the thumb MCPJ, it is evident that A\&E SHOs are unable to diagnose these injuries correctly. This implies that the threshold for referral should be lowered, so that all injuries around the MCPJ of the thumb are seen early by an appropriate specialist.

Pre-hospital ALS-the role of MEDIC 1 A P Gleeson

Accident and Emergency Department, Royal Infirmary of Edinburgh, Lauriston Place, Edinburgh

Objective-To assess the effectiveness of a land based flying squad (MEDIC 1 ) as a secondary response unit to victims of a cardiac arrest during a period when ambulance paramedics were using their full range of skills.

Patients and methods-Retrospective analysis of all cardiac arrests attended by MEDIC 1 in the Lothians and Borders Regions between 1993 and 1996.

Results-(see table 1 below): 145 patients were attended by MEDIC 1 and there were 20 survivors $(13.7 \%)$. All but two survivors had been converted to sinus rhythm by the ambulance crew before the arrival of the MEDIC 1 team. Interventions by MEDIC 1 on survivors were the administration of anti-arrhythmic drugs in two patients, intubation in four, and cardioversion in three.
Conclusions-A land based flying squad functioning as a secondary response to victims of cardiac arrests does not have a significant effect upon survival.

Vectorcardiography using the MIDA system: a new tool for the diagnosis and management of ischaemic heart disease in the A\&E department

$\mathrm{J}$ Webb, E Glucksman, R Brown

Department of Accident and Emergency Medicine, King's College Hospital, Denmark Hill, London SES

Myocardial infarct size and remaining myocardial ischaemia are recognised as the key determinants of survival following a myocardial infarct. Since the advent of thrombolysis in the management of myocardial infarction the morbidity and mortality have been significantly reduced. The earlier the infarct related vessel is reopened the better the prognosis. It is therefore important to be able to recognise reperfusion and consider angioplasty for those patients in whom reperfusion is not achieved. The MIDA system (Myocardial Ischaemia Dynamic Analysis, Hewlett Packard) uses non-invasive vectorcardiography to monito changes in the ST segment and QRS complex dynamically, allowing therapeutic decisions to made in real time. This is achieved by using surface electrodes which monitor vectors within the myocardium and transfer the information to a computer, where it is displayed graphically. Criteria have been established to indicate vessel patency following thrombolysis for myocardial infarction. These are a change in the QRS morphology, which reaches a plateau within $2 \mathrm{~h}$ of thrombolysis, and a decline in the magnitude of the ST vector of $50 \%$ within $90 \mathrm{~min}$ (sensitivity $94 \%$, specificity $80 \%$ ). Criteria have also been established for the diagnosis of ischaemia. Initial experience of a unique installation of a MIDA system in an $A \& E$ department will be presented $(22 \%$ of patients with myocardial infarction; $50 \%$ with myocardial ischaemia; $14 \%$ with extracardiac causes of chest pain, and $14 \%$ without chest pain) with regard to initial patient management.

\section{A prospective study of post-traumatic} stress disorder in A\&E attenders

$\mathrm{S}$ Mason

Accident and Emergency Department, Northern General Hospital, Herries Road, Sheffield

This prospective study recruited 203 consecutive male patients between the ages of 18 and 60 years admitted from the $\mathrm{A} \& \mathrm{E}$ department following accidental injury over a six month period. The aims of this study were to document the incidence of post-traumatic stress disorder among everyday attenders to

Table 1 the A\&E department. Using certain demographic details, information surrounding the accident itself, and standardised measures such as the Revised Impact of Events Scale, the study aims to identify simple ways of detecting post-traumatic stress disorder in this type of patient. Each patient had an initial interview and assessment by an A\&E research fellow while in hospital, and was then followed up at between four and six weeks after their injury, and again at six months post-injury. The follow up contact documented ongoing physical problems and reassessed psychological sequelae, using the Revised Impact of Events Scale and other standardised psychological measures. Results will present the frequency and degree of psychological disturbance that can be inflicted by everyday trauma. This may give some insight into the natural history of responses to traumatic stress and the pattern of reactions in patients following a normal recovery from injury.

How do individuals with diabetes use the accident and emergency department? E C Goyder', S W Goodacre ${ }^{2}$, J L Botha ${ }^{1}$, G Bodiwala ${ }^{3}$

Department of Epidemiology and Public Health, University of Leicester, ${ }^{2}$ York District Hospital, and ${ }^{3}$ Accident and Emergency Department, Leicester Royal Infirmary, Leicester

Previous studies have suggested that diabetic individuals, despite greater morbidity, attend the accident and emergency (A\&E) department no more often than the general population. This implies that they attend less than the general population for indications unrelated to diabetes and that they may use lay or primary care resources in situations where a non-diabetic individual would attend A\&E The aim of this historical cohort study was therefore to determine whether individuals with diabetes do have a different pattern of A\&E use to the general population. Service use by 696 individuals with diabetes over an 11 year period was compared with use by 696 non-diabetic individuals. The two cohorts were matched on age, sex, and general practitioner. The geographical distributions of their addresses were similar. More visits were made by the diabetic cohort $(1002 v 706, \mathbf{P}=$ $0.0001) .121$ visits by the diabetic cohort were directly related to diabetes, including 52 for hypoglycaemia. The diabetic cohort also had more visits for medical illness unrelated to diabetes (357 $v 231, P=0.0001)$. The number of visits for injuries, including self harm, was not significantly different $(524 v$ $475, P=0.3$ ). Individuals with diabetes who attended $\mathrm{A} \& \mathrm{E}$ were not significantly more likely to be referred by a general practitioner $(14 \% v 16 \%)$ or admitted $(20 \% v 17 \%)$. They were more likely to have arrived by ambulance ( $36 \% v 26 \%, \mathrm{P}=0.02$ ), even for injury related visits $(24 \% v 15 \%, P=0.05)$. Individuals with diabetes made more frequent visits than the general population to the $A \& E$ department. These excess visits were for both diabetes and other medical illnesses. Since there was no excess of visits for injuries, and the proportion requiring admission was similar, the difference is probably due to greater morbidity rather than a lower threshold for attendance.
VF/VT

Asystole

EMD ambulance ( $n$ ) Sinus rhythm on (n)

discharge

88
40
13
4

23
5
1
2

$\begin{array}{ll}34 & 18 \\ 10 & 0 \\ 2 & 1 \\ 1 & 1\end{array}$

,


Open water scuba diving accidents A J Hart, S A White, P J Conboy, G Bodiwala, D Quinton

A $\mathcal{E} E$ Department, Leicester Royal Infirmary, Leicester

Open water scuba diving is a dangerous sport. An audit was undertaken of diving accidents over a 5 year period presenting to the accident and emergency (A\&E) department of a large teaching hospital situated 12 miles from the largest inland diving centre in the United Kingdom. Between 1992 and 1996 the number of accident $(n=25)$ dramatically increased. Deaths $(n=6)$ increased threefold, and the number needing hospital admission for more than one day increased fivefold (range 1-30 days). The median depth of all accidents was 24 metres (range 7-36 metres), ascent profiles were rapid in $48 \%$, normal in $24 \%$, unknown in $28 \%$. The main factor for the increase in diving accidents is the increase in the number of dives undertaken, the overall incidence was 1 in 12000 dives. The increase in the number of dives is a result of technological, political, and social factors. We conclude that while everything possible is being done to reduce the complications of diving accidents at this institution, preventative safety awareness for divers needs to be improved and a regional hyperbaric unit should be set up.

\section{Predicting biphasic anaphylactic} reactions

A MacNamara, E Brazil

Accident and Emergency Department, The Leicester Royal Infirmary NHS Trust, Infirmary Square, Leicester

Objective-To assess how commonly biphasic anaphylactic reactions occur in patients admitted to a short stay $A \& E$ ward following treatment of an acute anaphylactic reaction. We analysed these cases to determine whether there were any markers that would allow early identification of patients who would subsequently develop a biphasic response. Design-Retrospective review of case notes. Subjects-34 patients admitted with anaphylaxis which required treatment with adrenaline.

Setting - Short stay ward of a medium sized A\&E department.

Results-Six patients (18\%) had biphasic reactions. No clinical features on initial presentation identified those likely to have a biphasic response. These patients, however, required significantly more adrenaline to ameliorate their initial symptoms $(P=0.03)$ than those having a uniphasic reaction.

Conclusions-Biphasic anaphylactic reactions occur frequently. There are no clinical features which allow identification of patients likely to have a biphasic response. These patients require higher doses of adrenaline to control their initial symptoms and this should be considered a marker for patients who may develop a biphasic response. These results confirm that all patients being discharged after treatment for an acute anaphylactic reaction must be made aware of the risk of a second phase response following apparent clinical resolution.
Sports hand injuries-an

epidemiological study

M Choyce, M Potts, A Maitra

Royal Victoria Infirmary, Newcastle upon Tyne

Sporting injuries are common in the developed world. Hand injuries are also a common and important source of incapacity and morbidity but have been relatively neglected in this context. We therefore decided to evaluate the nature and effects of hand injuries sustained during sport.

Methods-All patients presenting to the RVI A\&E department between 29 July 1995 and 28 July 1996 with a hand injury sustained during sporting activity and who received specialist follow up (by A\&E or plastic surgery units) were enrolled in the study. Additionally, patients were contacted at median of four months (range two to 13) after discharge for their assessment of outcome.

Results-262 cases were enrolled into the study. Follow up data were obtained by telephone in $206(79 \%)$, and by letter in a further $24(9 \%)$. Fractures were the commones injury (68\%), followed by soft tissue injuries $(20 \%)$ and dislocations (11\%); 45 cases $(17 \%)$ underwent manipulation and $15(6 \%)$ had surgery. There were highly significant differences between men and women, both in the ages affected and the type of sport resulting in injury to the hand. These patterns of injuries will be presented and discussed. Follow up indicates that mild impairment is common $(45 \%)$, while the incidence of moderate or serious problems was $10 \%$.

Conclusions-Sporting injuries to the hand are common. They may result in significant short term and longer term impairment of function. Telephone follow up of such injuries is a valid and acceptable way to obtain outcome data Certain sections of the population are at most risk of injury from a few specific sports. Thi study indicates suitable target areas for injury prevention.

\section{The management of an uncomplicated} traumatic pneumothorax

$S$ Mitra, T Soulsby

Accident and Emergency Department, Fazakerley Hospital, Liverpool

Objective-To establish current practice in the management of an uncomplicated traumatic pneumothorax.

Methods-A postal survey of 264 A\&E doctors given four case histories.

Results-Although many patients were observed, a high proportion was treated by intercostal drainage. Aspiration was also used in a significant proportion of the cases.

Conclusions-There is no clear consensus of opinion on the management of an uncomplicated traumatic pneumothorax. A randomised multicentred prospective trial is needed to clarify the situation.

\section{Medical hypnosis-a useful adjunct in paediatric suturing: randomised prospective controlled trial $\mathrm{H}$ Dowd \\ Department of Emergency Medicine, Cardiff Royal Infirmary, Newport Road, Cardiff}

Setting-A large emergency department suturing over 1200 children annually.

Subjects - children (age range 4-12) requiring sutures for wounds less than 6 hours old.
Exclusions-those with wounds $>6$ hours old admission for other injuries; those with fractures; those with head injuries/periods of unconsciousness; children on anticonvulsants, sedatives, or other drugs affecting cerebral function/cognition (including opiates and entonox); children who did not have their primary carer in attendance

Methods-Subjects were randomised into the study and control group. Children in the control group were sutured in the method standardised for the study (primary carer in attendance, paediatric nurse, warm lignocaine $\left(42^{\circ} \mathrm{C}\right), 24$ gauge needle, $3 \mathrm{~min}$ lag before suturing). Ethics approval was obtained.

Measurements-Glasgow coma scale, CHEOPS score (for sedation), ease of hypnosis.

Results-In all children who were successfully hypnotised, the level of activity was lower than in the controls. This allowed for ease of treatment In neither group was failure recorded (classified as child requiring pharmacological sedation or general anaesthetic). As an adjunct to the study, it was discovered that certain trigger words make children less susceptible to hypnosis. There was a distinct advantage to the use of the least emotive words, even as negatives.

Conclusions-Medical hypnosis has not previously been reported in paediatric emergency medicine in this country. This technique (which is relatively simple to learn) has many advantages and few drawbacks. It allows the child to use his imagination to create something pleasant rather than conjure up fear of the unknown in the suture room.

\section{A review of medicolegal surgical cases} which presented to UK accident and emergency departments

M Obiako, N L Jones, R J Evans, R C Evans Cardiff Royal Infirmary, Cardiff

Objective-To investigate the reasons for legal claims against UK accident and emergency departments in surgical cases.

Design-Retrospective analysis of requests for medical opinions submitted by solicitors between December 1989 and August 1995 on legal claims against UK accident and emergency departments.

Subjects-34 cases from the UK.

Outcome measures-The principal underlying causes of claims.

Results-The commonest reason for a claim was missed appendicitis (nine cases). Others included: six missed perforations: duodenal ulcers (3), diverticulum (1); duodenum(1) pancreatic duct (1); four medical causes: tuberculous meningitis (1), intra-abdominal tuberculosis (1), mesenteric adenitis (1) dissecting aneurysm (1); and two gynaecological causes: ectopic pregnancy (1), pyosalpinx (1). There were 13 others: ruptured Wilm's tumour (1); bruising secondary to venesection (1); infected IV insertion site (1); infected laceration to thigh (1); septicaemia secondary to renal calculus (1); intestinal obstruction (1); gangrenous toe (1); ruptured bladder (1) femoral artery embolus (1); infected haematoma (1); missed intra-abdominal foreign body (1); testicular torsion (1); missed ruptured abdominal aneurysm (1). In 11 cases, legal action was stimulated by comments made by a health care worker. The alleged causes were varied and included substandard history, substandard examination, insufficien investigation, and inadequate treatment. In 23 of the cases, the allegation was considered to be supportable. 
Conclusions - To reduce the incidence of errors, A\&E doctors should consult senior surgeons and relevant specialists about surgical cases which present to their departments.

\section{Value of blood cultures taken in the} emergency department

A-M Kelly

Emergency Department, Western Hospital, Private Bag, Footscray, Victoria 3011, Australia

Aims-(1) To determine whether blood cultures taken in the emergency department affect patient outcome; (2) to develop guidelines for the appropriate ordering of blood cultures in the emergency department.

Methods-Retrospective review of 1062 blood cultures taken in the emergency departmen of Western Hospital Footscray between 26/ $9 / 95$ and 30/6/96. These were classified and correlated according to diagnosis made in the emergency department. For those where a positive culture was received, case notes were reviewed to determine if it was a true or false positive and whether there was any impact on patient management.

Results-The number of true positive blood cultures was $56(5.2 \%)$. Of these, only 18 $(1.6 \%)$ affected patient management. The group where blood cultures had the greatest impact on patient care was in those patients diagnosed with generalised sepsis, in whom $5 \%$ of blood cultures directly influenced management.

Conclusions-Blood cultures taken in the emergency department rarely affect patien care. Where a "direct" specimen from the clinically suspected site of infection is possible (for example, cerebrospinal fluid, urine, join aspirate, pus from an abscess) this should be cultured instead of blood. Patients for whom clinical diagnosis of viraemia, minor infection or a non-infective condition is made should not have blood cultures performed. The performance of blood cultures should be reserved for patients considered to have major infections requiring hospital admission for whom direct specimens are not possible.

\section{Leeches loose in the accident and} emergency department

\section{Obiako}

Accident and Emergency Department, Cardiff Royal Infirmary, Cardiff

The medicinal leech (Hirudo medicinalis), has been used in medicine for centuries and has been enjoying a resurgence in popularity in recent years. Leech therapy is used mainly in plastic and reconstructive surgery units to improve the viability of skin flaps, replanted digits, and ears, and in the evacuation of haematomata around surgical wounds. We have used leeches successfully on three patients in this department to evacuate traumatic haematomata of the pinna, in an attempt to prevent the unsightly cauliflower ear so characteristic of rugby players. The only caution is the potential for infection with Aeromonas hydrophilia. This Gram negative bacillus is a symbiont in the leech digestive tract, and is essential for digestion of a blood meal, as the worm does not possess proteolytic enzymes. Aeromonas septicaemia is a rare complication of leech therapy and is prevented with prophylactic antibiotic treatment using Augmentin, the quinolones, or third generation cephalosporins.
Metal detector and swallowed metal foreign bodies

N V Doraiswamy, H Baig, L Hallam

Royal Hospital for Sick Children, Yorkhill NHS Trust, Glasgow

Aim-To confirm value of metal detectors in detecting metallic foreign bodies (MFB) and to compare the results with $x$ rays.

Materials and methods-231 children were brought to A\&E with a history of swallowing an MFB over 18 months in 1995-6. A meta detector with audiovisual signals (AVS) was used and was tested on a metal object before each examination. We recorded whether the history of swallowing was definite or only suspected. The neck, sternal region, and all quadrants of the abdomen were scanned for AVS and the results recorded. Subsequently, $x$ rays were taken and the findings compared. When $x$ ray findings were negative and the metal detector test positive, false positive were identified by a careful search for any previously unrecognised metallic objects in the child's environment.

Results-63\% of the cases were boys and 37\% girls. A definite history of swallowing an MFB was available in $186(81 \%)$, but three $(2 \%)$ proved negative for both the metal detecto test and $x$ rays. "Child was playing and the MFB is now missing" was the history obtained in the remaining 45 children (19\%) Four of these $(9 \%)$ were positive for both tests. AVS were obtained in $187(81 \%)$ and an MFB confirmed in the corresponding $x$ rays AVS were obtained in 112 cases $(48.5 \%)$ in left upper quadrant, in 20 cases $(8.7 \%)$ in the epigastrium, in 14 cases $(6.7 \%)$ in the sterna and high epigastric region, and in eight cases $(3.5 \%)$ in the neck or in the remainin segments of the abdomen. $X$ rays were positive in the corresponding regions. Recog nised causes of false positives were two children with sternotomy wiring and one mother wearing a gold ring while holding her child. Weak AVS were observed from a stee chair in two cases, and in three cases because of a zip, a metal button, and a coin in the trouser pocket, respectively. A repeat metal detector test after a negative $x$ ray was also negative after eliminating the extraneous cause. Al these false positives occurred in the earlier period of the study. There were no false negative results (that is, a negative metal detector test and a positive $x$ ray)

Conclusions-A metal detector is a useful alternate for $x$ rays whenever there is possibility of a swallowed MFB. It is cheap readily available, non-invasive, can be repeated instantly, and has no maintenance expenses. It avoids the radiation and cost of $x$ rays, and the inconvenience of waiting in overcrowded radiology departments. The technique can be learned easily and can be used by any medical or nursing personnel. The possibility of false positive should be borne in mind.

\section{Accident and emergency review} clinics-needless luxury or necessary evil? G I Quin, G M McCarthy

Accident and Emergency Department, Royal Gwent Hospital, Newport

It is widely held that a proportion of $A \& E$ patients will require review at some time after their initial attendance. In many departments much of this activity occurs in structured A\&E review clinics, while in others no such clinics are run, without apparent adverse effect. The aim of our study was to determine the benefits, or otherwise, of such a clinic-as described by both patients and cliniciansand to ascertain the views of the specialty as a whole on this subject.

Methods-Questionnaires were offered to all patients attending our $A \& E$ review clinic in November and December 1996, and senior $A \& E$ clinicians conducting the clinics were also asked to complete a questionnaire on each patient. In addition. we circulated a questionnaire to all $\mathrm{A} \& \mathrm{E}$ consultants in the UK and Republic of Ireland.

Results - The results of 412 patient and 739 clinician questionnaires are presented, showing that a majority of patients described their visit as useful $(95 \%)$ and convenient $(78 \%)$. Clinicians deemed $88 \%$ of patients to have been appropriately referred to the clinic, and in $92 \%$ the initial $\mathrm{A} \& \mathrm{E}$ management was described as appropriate A more detailed analysis of the background to these responses is presented. Results, to date, of the nationwide survey suggest that an overwhelming majority (97\%) of $A \& E$ consultants feel that review clinics are useful, although $20 \%$ expressed reservations. These issues are discussed further.

Conclusions-Patients expressed a high level of satisfaction with their experience of the review clinic. This response, coupled with that of the clinicians, suggests that the clinic is a useful component of our department's activity. There is a role for $A \& E$ review clinics within the specialty, although their precise place in terms of caseload, structure, and funding needs to be defined locally rather than on the basis of national consensus.

Head injury advice-worth the paper it's written on?

E Walker

Accident and Emergency Department, Dewsbury District Hospital, Healds Road, Dewsbury

In mid-1995, we decided to update the "head injury instructions" given out within our department. In order to facilitate this, and in an attempt to incorporate "best practice", I conducted a postal survey of every A\&E department in Great Britain at that time which involved sending out 273 letters (with a prepaid reply envelope) asking for a copy of that hospital's own head injury advice sheet. The address database was provided by the Nursing Development Unit within our department and the letter was addressed to th nurse in charge of the department concerned. We achieved a response rate of $81 \%$. The advice given was so disparate and of such varying quality, that I compiled the results onto a database, listing the frequency with which each piece of advice was mentioned This paper presents the results of this postal survey. The most commonly quoted warning symptom was "Severe headache" at $68.2 \%$, and the least common was "Difficulty swallowing", at $0.4 \%$. In between, there are 41 categories of warning signs, including nasa discharge, difficulty breathing, and abdominal pain. $24.9 \%$ of departments advise their patients to avoid heavy meals following a head injury, whereas $4.3 \%$ advise refraining from driving or operating machinery for 24 hours, and only $0.4 \%$ advise avoidance of contact sports for 2-4 weeks. While the prime motivation behind this survey was to obtain information for our own use, it quickly became 
apparent that some of the advice and information being given out was of very poor quality indeed. It would seem reasonable to expect that advice given to patients on detecting potentially fatal delayed brain injury might be roughly equivalent throughout the country. At the moment, this is very far from being so.

\section{Problems encountered in the urgent} transfer of trauma patients from peripheral hospitals to tertiary centres for specialist care in Northern Ireland

B S Sinnott, L G Rocke L G, R L S Spedding, J A Steele, B P McNicholl

Accident and Emergency Department, Belfast City Hospital, Belfast

Objective-To identify problems with the transfer for urgent trauma cases from peripheral $A \& E$ departments for specialist care in two tertiary referral centres.

Design-A prospective study from 15 peripheral $A \& E$ departments in Northern Ireland and two large teaching hospitals.

Subjects-All acute trauma patients presenting to the $A \& E$ departments of the peripheral hospitals from 1 March until 31 August 1995 who required transfer for life or limb threatening conditions.

Measured variables - Time spent in the primary hospital; the nature of the injuries; the number of telephone calls to the central hospital per patient; the number of specialists contacted; time necessary to secure agreement for the transfer; time required to secure transport (always by ambulance).

Results-56 patients were referred from 11 hospitals. The average time for initial stabilisation in the primary hospital was $71 \mathrm{~min}$ and for contacting and agreeing transfer with the receiving specialist was $19 \mathrm{~min}$. An average of 2.3 telephone calls were made for each patient. The average time to arrange and obtain transport was 61 minutes. Ten patients required more than one specialist contact in the tertiary centre. Seventeen of the patients were referred by senior house officers only. The average time per patient for initial stabilisation, to reach agreement on transfer, and arrange for transport to the tertiary centre was $153 \mathrm{~min}$.

Conclusions-Delay in contacting specialists and organising transport is prolonging patient transfer. Multiple telephone calls are required. Receiving hospitals should provide more rapid acceptance of transfers. A single trauma coordinator consisting of senior clinicians on a rota could do this.

\section{Psychological support for children after} a disaster

R B Leonard', R L Alson', C Mahan², L W Stringer ${ }^{3}$

'Department of Emergency Medicine, Bowman Gray School of Medicine, Winston-Salem; ${ }^{2}$ Brenner Children's Hospital, Bowman Gray School of Medicine, Winston-Salem; ${ }^{3}$ Special Operations Response Team, Winston-Salem, North Carolina, USA

Disasters come in many forms with a number of them causing great destruction to an entire community or region. In addition to causing death and injuries, families can be left without their homes and possessions. The initial relief effort of response personnel who respond is to attend to the medical needs of citizens involved, followed by supplying food and shelter. In the days that follow, one topic that can be easily overlooked is the psychological support of the victims, especially the children. The psyche of children can be devastated by loss of their home, toys, pets, and play groups. Parents, also affected by the disaster, may well be physically and emotionally unable to attend to the needs of their children. We will describe some simple materials for a disaster team to have that are aimed at the psychological support of children affected by a disaster. Disaster response teams are usually comprised of people with medical training as opposed to training in psychology and psychiatry. Accordingly, the materials with which they are supplied are simple and contain a brief explanation as to their use. Play therapy and art therapy, as well as different levels of games, can be put to great use in the psychological support of children. The disaster team is also supplied with a 20 page summary of the abnormal behaviour patterns likely to be seen in children in response to the stressors of a disaster. The goal is not to become a baby sitting service, but rather to offer useful help in dealing with the psychological problems of children in a stressful situation.

\section{Efficacy of a charcoal delivery system}

R Boyd

Accident and Emergency Department, Royal Preston Hospitals, Sharoe Green Lane, Fulwood, Preston, Lancashire

Aims - To ascertain the actual amount of activated charcoal ingested compared to that prescribed by medical staff through both oral and orogastric routes, and to ascertain the frequency of vomiting in the hour after charcoal administration, by either route.

Methods-A cohort of 100 consecutive administrations of charcoal to patients $>16$ years of age was followed prospectively. All prescribed doses of charcoal were made up to a presentation volume of $400 \mathrm{ml}$ in a translucent vinyl container. The mixture was agitated every 5 min. Ingestion time was $30 \mathrm{~min}$. The residual volume left was estimated using graduated markings on the presentation container. A history of vomiting was elicited for the hour following ingestion.

Results-Full data were collected for 76 patients. Of these, $74 \%$ were given charcoal orally. $39 \%$ of orally prescribed doses resulted in ingestion of $<70 \%$ of prescribed dose; $27 \%$ of orogastric prescribed doses resulted in < $70 \%$ ingestion of the prescribed dose. $29 \%$ of patients given charcoal orally vomited within $1 \mathrm{~h}$ of charcoal administration; $37 \%$ of patients given charcoal orogastrically vomited within $1 \mathrm{~h}$ of administration (NS)

Conclusions - The use of activated charcoal is now well established; however, there are significant problems with administration and with postingestion vomiting with the most common forms of delivery.

Northern Ireland's riot week 1996: plastic bullet injuries

S J McBride, J Steele, J Kelly, C Dearden Mater Hospital Belfast, Accident and Emergency Department, Crumlin Road, Belfast

Controversy has attended the use of plastic bullets right from their first use in the UK Plastic bullets have been available to police forces as a method of riot control throughout the UK since 1988, but have been exclusively used in Northern Ireland by the security forces since their introduction in 1973, completely replacing the less accurate rubber bullet in 1975. Some 17 fatalities have been attributed to plastic bullet use. The plastic bullet is a projectile made of polyvinyl chloride
(PVC). It is a cylinder measuring $10(3.7 \mathrm{~cm}$ and weighs $13.5 \mathrm{~g}$. It is discharged from the riot control gun at low muzzle velocity to travel on its long axis to hit the target end on. During the week of 8-15 July 1996 (the peak of the annual Orange "marching season") widespread serious public disorder occurred in Northern Ireland and some 6000 plastic bullets were fired. We report a series of 177 patients who attended $A \& E$ departments in eight hospitals (seven in Northern Ireland and one in the Irish Republic) during this period. For 176 of these patients 183 separate injuries attributed to plastic bullets have been recorded. There were 39 injuries to head and neck, 31 to upper limb, 26 to chest, 13 to abdomen, and 74 to lower limb. Injury severity scores were calculated for these 176 patients. The scores ranged from 1 to 18 . A total of 45 patients required hospital admission, of whom four required intensive care treatment. There were no fatalities.

One year's experience of outpatient treatment of deep vein thrombosis D F O'Shaughnessy', C Tovey ${ }^{3}$, S Akbar ${ }^{2}$, A L Miller ${ }^{1}$, P B Rana', V O'Neill', C A Dinneen ${ }^{1}$

Departments of 'Haematology and ${ }^{2}$ Accident and Emergency, St Peter's Hospital NHS Trust, Guildford Road, Chertsey, Surrey; ${ }^{3}$ The Accident and Emergency Department, The Conquest Hospital, Hastings, Sussex

The aim of this pilot study was to assess whether patients with acute deep vein thrombosis (DVT) could be satisfactorily treated on an outpatient basis with low molecular weight heparin (LMWH) and warfarin. 635 patients with a suspected DVT seen by their GPs were referred to accident and emergency and assessed by A\&E staff. They were then referred for light reflectance rheography (LRR). 205 patients with a positive LRR, or a negative LRR and strong clinical suspicion, underwent venography: 95 were venogram positive, of whom 91 , between the ages of 22 and 89 years, have now been treated with LMWH as outpatients. Eleven had carcinoma as an underlying cause, of whom eight have now died, and four had a thrombophilia; the remainder $(83 \%)$ were classed as idiopathic. None had further embolic events. Two had minor bleeding (when on warfarin), but none had major bleeding. The costs of this treatment compare favourably with the costs of continuous intravenous heparin-the previous treatment used-which needs to be given daily by intravenous pump and has to be monitored by daily activated partial thromboplastin time measurements. Direct liaison with community nurses has minimised the impact on GP workload. 728 bed-days were saved during this period by outpatient treatment of DVT, an estimated cost saving of $£ 183300$. We feel in the near future the outpatient treatment of DVT will be standard procedure and the treatment of pulmonary thromboembolism will soon benefit from shortened inpatient stay (or even outpatient treatment).

\section{Use of electronic ear syringe in the} emergency department

I Jones

Salford, Lancs

The removal of foreign bodies from the external acoustic canal is a common problem, especially in children. Classic techniques with Hartmann forceps and loops can be traumatic. Suction is 
rarely available, requiring specialist referral. Syringing is an accepted practice for the removal of wax, which is regularly performed in general practice. An electronic ear syringe allows easy removal of non-hygroscopic foreign bodies in the emergency department. The device is cheap, easy to use, and produces a rapid, effective treatment, with good patient compliance. The propulse ear syringe has been used for two years in bolton, without problems and proved cost-effective. In view of increasing patient numbers and demands, such a device can assist in improving patient satisfaction and flow in the emergency department.

Change in frequency of fractures during puberty

Z A Ralis, P W Richmond

Accident and Emergency Department, Bone Research Laboratory, Cardiff Royal Infirmary, Newport Road, Cardiff

It is generally accepted that following the menopause, the decreased levels of circulating sex hormones are responsible for the increased fragility of aging bones, resulting in a dramatic increase in the number of patients with "osteoporotic" fractures of the forearm, vertebral body, femoral neck, upper humerus, and pelvis. Oestrogens and testosterone have a beneficial effect on the skeleton, as shown by their preventative and therapeutic effects after the menopause, in hypogonadism, and after ovariectomy. We investigated whether the beneficial effects of sex hormones on bone tissue could be demonstrated at the other end of sexual maturity, that is, at puberty. All patients aged 7-20 years who attended the $A \& E$ department at Cardiff Royal Infirmary over two years 1995-1996 with a radiologically confirmed fracture of the lower end, or shaft, of the forearm were studied. The frequency of both lower forearm and forearm shaft fractures (number of fractures per 100000 of population of the given age and sex) was studied separately in both sexes for each year of their age. Lower end and shaft fractures of the forearm show a significant decline in the fracture frequency associated with the onset of sexual maturation, that is, in girls at the age of 11-12 years and in boys at the age of 13-14. The decline in the frequency of fractures, often 3-5 times lower than before puberty, continues into young adulthood. This study indicates for the first time that the mechanical competence of bones is positively influenced by the sex hormones from the very beginning of sexual maturity. Possible mechanisms are discussed.

\section{Childhood fracture surveillance-a} geographical study

A M Dielahunty', D Kraus', H Allen², M McCabe ${ }^{2}, \mathrm{P} \mathrm{Nash}^{3}$, R A Lyons

Morgannwg Health Authority, Swansea $;{ }^{2}$ Morriston

Hospital, Swansea; ${ }^{3}$ Neath General Hospital, Neath

Aim-To identify factors involved in causing childhood fracture.

Methods-For every child aged less than 17 years (or parent, as appropriate) who attended $\mathrm{A} \& \mathrm{E}$ with a new fracture, information was sought by the diagnosing doctor concerning the location of the event, the activity at the time of injury, the mechanism of injury, and any other related factors. Computerisation of both $A \& E$ departments serving the district population allowed identification of all cases, and follow up by phone or letter where information was incomplete. Data were coded using the NOMESCO system.
Results-In the first six months, 1465 fractures occurred, a rate of 84.3 per 1000 children aged $0-16$ years. Two thirds were in boys, $92 \%$ caused by accidental injury and $6.6 \%$ by violence. Of accidental injuries, $14 \%$ occurred in the home, $11 \%$ in gardens, $21 \%$ at school, $12 \%$ on sports fields, and $6 \%$ in sports halls; $8 \%$ occurred in public playgrounds; $42 \%$ of cases were involved in active leisure or sport at the time of the injury, $40 \%$ of which was organised. One third (69) of organised sports fractures were sustained playing rugby, one sixth (35) playing football, and $8 \%(18)$ in combat sports. The commonest nonorganised "sport" fractures were from cycling (87), football (79), roller blading/ skateboarding (60), and rugby (14); 89 children were injured on playground equipment; $1.5 \%$ of children sustained their injury on the roads, 10 as pedestrians, five as cyclists, four on motorbikes, and one in a car.

\section{Communication of A\&E deaths to GPs}

A Macleod, A Maitra

Accident and Emergency Department, Royal Victoria Infirmary, Newcastle upon Tyne

Communication between $A \& E$ departments and GPs is often difficult, because of the large numbers of patients passing through the department. SHOs' patterns of work mean that they will often not be available during GP surgery hours to communicate with a patient's own GP. When someone dies unexpectedly in the A\&E department it is particularly important that the GP is informed promptly so that a bereavement visit can be arranged. We decided to tackle this problem by finding out what type of communication local GPs expect of us, basing our departmental guidelines on their response.

Methods - 100 Newcastle GPs were randomly selected and sent a postal questionnaire.

Results-97/100 questionnaires were received back. Most GPs wanted to be informed of a patient's death within $24 \mathrm{~h}(88 \%)$. Most wanted to be informed by telephone, with confirmation in writing $(65 \%)$. Just over half of our local GPs $(52 \%)$ were happy for either doctor, nurse, or receptionist to inform them; $25 \%$ felt that it should only be a doctor who carries out this role; $79 \%$ of GPs were happy for a message to be left with the receptionist, and only $24 \%$ wanted their surgery to be interrupted to inform them of a death. We explored the possibility of using a fax machine to inform GPs of patient deaths. Although $93 \%$ had fax machines, $35 \%$ saw problems with using the fax for this purpose-the main potential difficulty being confidentiality. Conclusions-Communication between A\&E and GPs can be difficult. We have used a questionnaire to find out what GPs actually want and are proposing to change our practice in the RVI department.

Is single breath counting an alternative to peak flow in the assessment of acute asthma?

A Cooper

Accident and Emergency Department, Norfolk and Norwich Hospital, Brunswick Road, Norwich

Background-The ability to talk in sentences is often recorded as part of a clinical assessment of acute asthma. Single breath counting (SBC) is an objective method of quantifying the ability to talk. In a pulmonary function laboratory SBC has been shown to correlate with peak expiratory flow (PEF) and has been advocated as a "reasonable alternative" to PEF in the assessment of patients with exacerbations of asthma in the emergency department.

Objectives-This study was designed to assess whether SBC is a reliable alternative to peak flow in the assessment of asthmatic patients presenting to an $\mathrm{A} \& \mathrm{E}$ department.

Methods-The subjects were previously diagnosed asthmatics presenting to $A \& E$ with a PEF $<75 \%$ of best or predicted. Patients with other respiratory diagnoses and patients unable to talk or blow into a PEF meter were excluded. PEF and PEF\% (PEF/best PEF) were recorded. The assessing doctor gave each patient a demonstration of SBC and then asked the patient to do it. The number that the patient was able to count to before having to take another breath was recorded.

Results - 69 patients were enrolled in the study (39 male, 30 female). The range of presenting PEF was $100-410$ litres/min. Both variables were normally distributed. SBC correlated significantly with PEF ( $r=0.39 ; \mathrm{P}<0.01)$. There was no significant correlation with PEF\%. The technique of SBC and the clinical limitations of this correlation will be discussed.

\section{An SHO handbook of A\&E medicine} "breaking new ground"

P Bradley, A Shenton, M Smith

Bradford Royal Infirmary, Bradford

A recent survey of senior house officers in A\&E has revealed an urgent need for an innovative user-friendly pocket sized $A \& E$ handbook that addresses the needs of SHOs. To tackle this problem we have produced a computer generated pocket sized handbook, written from a senior house officer's perspective, which provides extensive coverage of the management of all the common A\&E conditions. One of its unique features is that it is computer generated and stored on diskette, so that SHOs can easily reprint and replace soiled or damaged pages in the ring bound handbook. Furthermore, the guidelines and management plans can easily be altered or adapted to meet changing local protocols. As the booklet is held on diskette it is easy to produce updated revisions, and this is done on an annual basis. To complement the handbook the department has also been extending its ranges of videos of minor procedures which are held on CDROM. This innovative dynamic computer generated education package for SHOs ensures a uniform provision of a high standard of care for patients and helps SHOs develop the confidence in managing the wide variety of conditions to which they have previously had little exposure.

\section{Care of "special needs" populations} during disasters

R L Alson', R B Leonard ${ }^{1}$, L W Stringer ${ }^{2}$

'Department of Emergency Medicine, Bowman Gray School of Medicine, Winston-Salem; ${ }^{2}$ Special Operations Response Team, 1728 S Hawthorne Road, Winston-Salem, North Carolina, USA

Among the tasks facing emergency personnel following a major disaster is the provision of shelter for evacuees. In most situations, sheltering consists of the provision of food, 
water, and protection from the environment. Medical care at most shelter facilities is usually limited to a "basic" level and sheltered persons are responsible for their own basic care and hygiene. The rise in the mean age of the US population and the changing health care environment has resulted in a change in the way health care is delivered. More persons reside in nursing homes or other domiciliary care facilities and increasing numbers of patients receive complex medical care at home, including intravenous therapy and home oxygen or ventilators. Conventional disaster shelters lack the necessary supplies and personnel to care for these "special needs", patients who are defined as those who need intermediate or skilled nursing care for management of chronic or acute diseases. Previously, such patients were evacuated to a hospital until the disaster was over. Current use of this approach would quickly overwhelm those medical facilities that have survived the disaster and deplete resources needed to care for victims of acute injuries and illness. We have developed a systematic programme to address the care of "special needs" populations in a disaster. A unique aspect of this programme is that it unites both emergency response agencies and private sector providers (nursing homes, home care, and medical sup ply companies) in a coordinated response to address the needs of this population. Identification of those populations at risk, resources in the community, and coordination of response are reviewed. Guidelines for implementation of such a programme will be presented.

\section{Effect of a large sports tournament on A\&E attendances}

$\mathrm{M}$ W Cooke ${ }^{12}$, Euro 96 Study Group of A\&E Departments, $S$ Wilson ${ }^{2}$, T Allen ${ }^{2}$

' AE'E Department, City Hospital, Dudley Road, Birmingham; ${ }^{2}$ Department of General Practice, University of Birmingham

Before the Euro96 football championships there was much discussion in the medical press of the effects of the multicentre tournament on $\mathrm{A} \& \mathrm{E}$ attendances. A\&E departments were concerned about the increased workload with no increase in funding. It was postulated that centres near stadia may see an increase in workload both from match violence and from the temporary increased local population. Aim - To determine any changes in attendances at $A \& E$ departments related to Euro96 match dates and during the tournaments. To determine if changes occurred at $A \& E$ departments close to match stadia and/or distant from them.

Methods-Analysis of attendance figures for three weeks preceding the tournament and during the tournament. Attendance in this study was defined as total new attendances to $A \& E$, excluding GP admission referrals. The relation between daily attendance rates and local matches was assessed by use of a generalised linear model, while taking into account the underlying trends in attendance rates including the usual variability observed by day of the week.

Results-No association was shown between number of attendances at $A \& E$, either near to or distant from matches. The occurrence of home nation (England or Scotland) matches had no correlation with attendance numbers. The only independent predictor of variation observed was day of the week, as has been previously noted.

Conclusions-These results do not support the hypothesis that large sports tournaments increase the number of $A \& E$ attenders. Although there are attenders from the matches and increased population, these maybe compensated for by local people remaining at home and therefore not suffering so many accidents and injuries. Further work is needed to analyse whether there is a change in case mix.

\section{Emergency Medicine March 1997 Contents}

\section{Editorial}

\section{Original research}

5 The incidence, severity and nature of violent incidents in the emergency department fennifer G Brookes, Robert $\mathcal{F}$ Dunn

11 The medical consequences of a golden oldies rugby tournament Michael Ardagh

\section{Case report}

15 Urethral self-insertion: case report and review of the literature Paul Lamberth

Technology

21 Helical CT in the diagnosis of pulmonary thromboembolism Niall Small

\section{Toxicology}

25 Nicotiana glauca toxicity Mark Webb, Shona Dalzell

29 Gamma hydroxy butyrate overdose Michael Ragg

\section{Therapeutic review}

32 Good outcomes using mask continuous positive airway pressure in elderly patients with acute pulmonary oedema Anne-Maree Kelly, Con Georgakas, Shan Bau, Pamela Rosengarten

\section{Review articles}

35 Vagal manoeuvres for termination paroxysmal supraventricular tachycardias Anne-Maree Kelly, Marie Kuhn 42 Diving emergencies David Smart

\section{Education}

45 Evolution and quality assurance of the primary examination of the Australasian College for Emergency Medicine Pamela Rosengarten, Anne-Maree Kelly Viewpoint

50 A history of triage in civilian hospitals in Australia Edward Brentnall

Book review

55 Primary care orthopaedics Sashi Kumar

56 Abstracts of the Annual Scientific Meetings of the ACEM and ASEM 1996

College news

76 President's message Christopher Baggoley

77 ACEM Office Bearers 1996/7

78 Board of Censors News Paul Mark

\section{Society news}

80 President's message Keith Edwards

81 ASEM Office Bearers 1996/7

\section{Letters to the editor}

82 The changing pattern of self-poisoning in Hong Kong over nine years Steven Doherty In reply Fei Lung Lau, Rico Liu, Sai Hang Yip

84 Noticeboard

87 Journal search

Information for contributors 